\title{
FUNCTION SPACES AND LOCAL CHARACTERS OF TOPOLOGICAL SPACES
}

\author{
TOSHIJI TEREDA
}

(Communicated by Dennis Burke)

\begin{abstract}
We write $V \simeq W$ to mean that the two linear topological spaces $V$ and $W$ are linearly homeomorphic. In this paper we prove: (1) There are compact spaces $X, Y$ for which $C_{p}(X) \simeq C_{p}(Y)$ and $\chi(X) \neq \chi(Y)$ are satisfied. (2) For each infinite cardinal $\kappa$, there are spaces $X, Y$ for which $C_{p}(X) \simeq C_{p}(Y), \chi(X)=\omega$ and $\psi(Y)=\kappa$. (3) For each infinite cardinal $\kappa$, there are spaces $X, Y$ for which $C_{p}(X) \simeq C_{p}(Y), \pi_{\chi}(X)=\omega$ and $\pi_{\chi}(Y)=\kappa$.
\end{abstract}

All topological spaces considered here are Tychonoff spaces. For an arbitrary topological space $X$, the space $C_{p}(X)\left(C_{s}(X)\right.$ in [2], $C_{\pi}(X)$ in [4]) is the set of all real-valued continuous functions on $X$ with the topology of pointwise convergence. The space $C_{p}(X)$ is a linear topological space under the algebraic operations being defined pointwise. We write $V \simeq W$ if two linear topological spaces $V$ and $W$ are linearly homeomorphic. Topological spaces $X, Y$ are said to be l-equivalent [1] if $C_{p}(X) \simeq C_{p}(Y)$. The weak dual of $C_{p}(X)$ is denoted by $L_{p}(X)$. It is well known that $C_{p}(X) \simeq C_{p}(Y)$ if and only if $L_{p}(X) \simeq L_{p}(Y)[\mathbf{5}, \mathbf{6}]$. Further, each topological space $X$ is embedded in $L_{p}(X)$ as a Hamel basis. Hence, if compact spaces $X$ and $Y$ are $l$-equivalent, then $\chi(Y) \leq\left|L_{p}(X)\right| \leq\left|R^{\omega} \times X^{\omega}\right| \leq 2^{\omega} \cdot 2^{\chi(X)}$.

In this note we will establish the following results.

EXAMPLE 1. There are $l$-equivalent compact spaces $X$ and $Y$ for which $\chi(X)=$ $\omega$ and $\chi(Y)=2^{\omega}$ are satisfied.

EXAMPLE 2. For each infinite cardinal $\kappa$, there are $l$-equivalent countably compact normal spaces $X$ and $Y$ for which $\chi(X)=\psi(X)=\omega$ and $\chi(Y)=\psi(Y)=\kappa$ are satisfied.

EXAMPLE 3. For each infinite cardinal $\kappa$, there are l-equivalent topological spaces $X$ and $Y$ for which $\pi_{\chi}(X)=\omega$ and $\pi_{\chi}(Y)=\kappa$ are satisfied.

Let $Y$ be a subspace of a topological space $X$. We define $C_{p}(X ; Y)=\{f \in$ $\left.C_{p}(X): f(Y)=\{0\}\right\}$. Pavlovskii [5] showed the following lemma.

LEMMA 1. If $Y$ is a retract of a space $X$, then $C_{p}(X) \simeq C_{p}(Y) \times C_{p}(X ; Y)$.

For a closed subset $Y$ of a normal space $X$, let $X / Y$ be the quotient space of $X$ obtained by collapsing $Y$ to one point $*$. In this case, the following lemma is a tedious exercise.

LEMMA 2. $C_{p}(X ; Y) \simeq C_{p}(X / Y ; *)$.

Received by the editors December 30, 1985 and, in revised form, October 8, 1986.

1980 Mathematics Subject Classification (1985 Revision). Primary 54C30; Secondary 46E10.

Key words and phrases. Real-valued continuous function, linear homeomorphism, character, pseudocharacter, $\pi$-character. 
1. Example 1. Let us recall the Alexandroff double circle $C_{1} \cup C_{2}$ [3]. The underlying set of this compact space is the union of two concentric circles $C_{i}=$ $\left\{(x, y) \in R^{2}: x^{2}+y^{2}=i\right\}$, where $i=1,2$. The subspace $C_{1}$ is the unit circle $S^{1}$ with its natural topology and the subspace $C_{2}$ is a discrete space of cardinality $2^{\omega}$. Obviously $C_{1}$ is a retract of $C_{1} \cup C_{2}$.

ClaIM 1. Let $A\left(2^{\omega}\right)$ be the one-point compactification of a discrete space of cardinality $2^{\omega}$. Then $C_{p}\left(S^{1} \oplus A\left(2^{\omega}\right)\right) \simeq C_{p}\left(C_{1} \cup C_{2}\right)$.

PROOF. Let $a$ be a point of $C_{2}$. Then

$$
\begin{aligned}
C_{p}\left(C_{1} \cup C_{2}\right) & \simeq C_{p}\left(C_{1} \cup\left(C_{2}-\{a\}\right)\right) \times C_{p}(\{a\}) \\
& \simeq C_{p}\left(C_{1}\right) \times C_{p}\left(C_{1} \cup\left(C_{2}-\{a\}\right) ; C_{1}\right) \times R \\
& \simeq C_{p}\left(C_{1}\right) \times C_{p}\left(\left(C_{1} \cup\left(C_{2}-\{a\}\right)\right) / C_{1} ; *\right) \times R \\
& \simeq C_{p}\left(C_{1}\right) \times C_{p}\left(\left(C_{1} \cup\left(C_{2}-\{a\}\right)\right) / C_{1}\right) \\
& \simeq C_{p}\left(S^{1}\right) \times C_{p}\left(A\left(2^{\omega}\right)\right) \simeq C_{p}\left(S^{1} \oplus A\left(2^{\omega}\right)\right) .
\end{aligned}
$$

Since $\chi\left(C_{1} \cup C_{2}\right)=\omega$ and $\chi\left(S^{1} \oplus A\left(2^{\omega}\right)\right)=2^{\omega}$, it follows that the desired example is obtained.

2. Example 2. For each infinite cardinal $\kappa$, let $W(\kappa+1)$ be the space of all ordinals less than $\kappa+1$ with the usual interval topology. Let $V(\kappa+1)$ be the subspace of $W(\kappa+1)$ consisting of all ordinals whose cofinality is less than $\omega_{1}$.

LEMMA 3. Let $F(\kappa+1)$ be the subspace of all limit points in $V(\kappa+1)$. Then $F(\kappa+1)$ is a retract of $V(\kappa+1)$.

ProOF. For each $\alpha$ in $V(\kappa+1)$, let

$$
r(\alpha)=\min \{\beta: \alpha \leq \beta, \beta \in F(\kappa+1)\} .
$$

Then $r: V(\kappa+1) \rightarrow F(\kappa+1)$ is a retraction.

Claim 2. Let $A(\kappa)$ be the one-point compactification of a discrete space of cardinality $\kappa$. Then $C_{p}(A(\kappa) \oplus F(\kappa+1)) \simeq C_{p}(V(\kappa+1))$.

PROOF.

$$
\begin{aligned}
C_{p}(V(\kappa+1)) & \simeq R \times C_{p}(V(\kappa+1)) \\
& \simeq R \times C_{p}(V(\kappa+1) ; F(\kappa+1)) \times C_{p}(F(\kappa+1)) \\
& \simeq R \times C_{p}(V(\kappa+1) / F(\kappa+1) ; *) \times C_{p}(F(\kappa+1)) \\
& \simeq C_{p}(V(\kappa+1) / F(\kappa+1)) \times C_{p}(F(\kappa+1)) \\
& \simeq C_{p}((V(\kappa+1) / F(\kappa+1)) \oplus F(\kappa+1)) .
\end{aligned}
$$

Since $V(\kappa+1)$ is countably compact, the quotient space $V(\kappa+1) / F(\kappa+1)$ must be homeomorphic with the space $A(\kappa)$. Hence

$$
C_{p}(V(\kappa+1)) \simeq C_{p}(A(\kappa) \oplus F(\kappa+1))
$$

It is obvious that

$$
\begin{aligned}
\chi(V(\kappa+1)) & =\psi(V(\kappa+1))=\omega, \\
\chi(A(\kappa) \oplus F(\kappa+1)) & =\psi(A(\kappa) \oplus F(\kappa+1))=\kappa .
\end{aligned}
$$

3. Example 3. First consider regular cardinals. For each infinite regular cardinal $\lambda$, let $U(\lambda+1)$ be the subspace of $W(\lambda+1)$ consisting of all ordinals $\alpha$ such that $\operatorname{cf}(\alpha) \leq \omega$ or $\alpha=\lambda$. 
CLAIM 3. $C_{p}(U(\lambda+1)) \simeq C_{p}(U(\lambda+1) /\{\omega, \lambda\})$.

PROOF.

$$
\begin{aligned}
C_{p}(U(\lambda+1)) & \simeq C_{p}(U(\lambda+1) ;\{\omega, \lambda\}) \times C_{p}(\{\omega, \lambda\}) \\
& \simeq C_{p}(U(\lambda+1) /\{\omega, \lambda\} ; *) \times R^{2} \\
& \simeq C_{p}(U(\lambda+1) /\{\omega, \lambda\}) \times R \\
& \simeq C_{p}(U(\lambda+1) /\{\omega, \lambda\}) .
\end{aligned}
$$

Note that $\pi_{\chi}(U(\lambda+1))=\lambda, \pi_{\chi}(U(\lambda+1) /\{\omega, \lambda\})=\omega$. Now consider a singular cardinal $\kappa$ and let $\left\{\lambda_{\alpha}: \alpha<\operatorname{cf}(\kappa)\right\}$ be a transfinite sequence of regular cardinals such that

$$
\sup \left\{\lambda_{\alpha}: \alpha<\operatorname{cf}(\kappa)\right\}=\kappa .
$$

Let $T(\kappa)$ be the topological sum of $\left\{U\left(\lambda_{\alpha}+1\right): \alpha<\operatorname{cf}(\kappa)\right\}$, and let $S(\kappa)$ be the topological sum of $\left\{U\left(\lambda_{\alpha}+1\right) /\left\{\omega, \lambda_{\alpha}\right\}: \alpha<\operatorname{cf}(\kappa)\right\}$. Then, by Theorem 2.6 in [4],

$$
C_{p}(T(\kappa)) \simeq C_{p}(S(\kappa)) \text {. }
$$

Further, it is obvious that $\pi_{\chi}(T(\kappa))=\kappa, \pi_{\chi}(S(\kappa))=\omega$.

\section{BIBLIOGRAPHY}

1. A. V. Arhangel'skii, Relations among the invariants of topological groups and their subspaces, Russian Math. Surveys 35 (1980), 1-23.

2. H. Buchwalter and J. Schmets, Sur quelques propriétés de l'espace $C_{s}(T)$, J. Math. Pures Appl. 52 (1973), 337-352.

3. R. Engelking, General topology, PWN, Warszawa, 1977.

4. D. J. Lutzer and R. A. McCoy, Category in function spaces. I, Pacific J. Math. 90 (1980), 145-168.

5. D. S. Pavlovskii, On spaces of continuous functions, Soviet Math. Dokl. 22 (1980), 34-37.

6. V. G. Pestov, The coincidence of the dimension $\operatorname{dim}$ of $l$-equivalent topological spaces, Soviet Math. Dokl. 26 (1982), 380-383.

Department of MATHEMATICS, FaCUlty OF ENgineERING, Yokohama National UNIVERSITY, 156, HODOGAYA, YOKOHAMA, JAPAN 